\title{
Systemic Capillary Leak Syndrome
}

\author{
Ruri Chihara, Hidetomo Nakamoto, Hiroshi Arima, Kenshi Moriwaki, Yoshihiko Kanno, \\ Soichi Sugahara, Hirokazu OKada and Hiromichi SuzukI
}

\begin{abstract}
Systemic capillary leak syndrome (SCLS) is characterized by recurrent hypovolemic shock attributable to increased systemic capillary leakage. A 46-year-old man was admitted to our hospital because of recurrent episodes of generalized edema with hypovolemic shock. Blood laboratory data revealed severe hypoproteinemia with a small monoclonal IgG- $\kappa$ protein. These findings suggested strongly the diagnosis of SCLS. A regimen with steroid pulse therapy was tried; however, intravascular overloading accompanied by the recruitment of the initially extravasated fluids resulted in acute pulmonary edema. Intensive care and careful monitoring of fluid volume are required in SCLS.
\end{abstract}

(Internal Medicine 41: 953-956, 2002)

Key words: shock, edema, hypoalbuminemia, pulmonary edema

\section{Introduction}

Systemic capillary leak syndrome (SCLS) was first reported by Clarkson and colleagues (1) in 1960. The syndrome is characterized by chronic recurrent episodes of a triad of hypotension, hypoalbuminemia and hemoconcentration associated with paraproteinemia (2). Rare complications of SCLS are renal damage and rhabdomyolysis, attributable to increased compartment pressure and ischemic myonecrosis (3). The degree of hemoconcentration relates to the overall severity of the acute phase of SCLS. SCLS often presents with an associated monoclonal gammopathy without any evidence of multiple myeloma or amyloidosis. The IgG class monoclonal gammopathy predominates, with either $\kappa$ or $\lambda$ light chains. Although more than seventy cases have been reported the pathogenesis of this syndrome remains unclear.

We describe a case of SCLS, with recovery from severe hypovolemic shock accompanied by pulmonary edema, and review the literature, with particular regard to the pathophysi- ology and the treatment of the syndrome.

For editorial comment, see $\mathrm{p} 909$.

\section{Case Report}

A 46-year-old man was admitted to our hospital because of generalized edema and disturbance of consciousness. In March 1999 , at the age of 45 , the previously healthy man had a sore throat, cough, and fever for several days. One month later, puffiness of the face and leg edema developed and required clinical evaluation elsewhere. His body weight rose from $60 \mathrm{~kg}$ to 64 $\mathrm{kg}$. On June 15, 1999, he was admitted to another hospital. Blood laboratory data revealed hypoproteinemia: total protein $5.6 \mathrm{~g} / \mathrm{dl}$, serum albumin $3.6 \mathrm{~g} / \mathrm{dl}$. A chest radiograph showed bilateral, slight pleural effusions. Common causes of generalized edema accompanied by hypoproteinemia, such as nephrotic syndrome, liver dysfunction, congestive heart failure and gastrointestinal protein losing enteropathy, were excluded. Twenty $\mathrm{mg} /$ day doses of loop diuretics were started and the generalized edema gradually disappeared. On July 10 , he was discharged from the hospital. One-week later, generalized edema and general fatigue returned, accompanied by nausea. He complained of excessive sweating and shortness of breath. On July 19, 9 days after discharge, he was brought to our hospital because of generalized edema and disturbance of consciousness (Fig. 1). The initial examination disclosed the following values: body weight $71 \mathrm{~kg}$, temperature $34.6^{\circ} \mathrm{C}$, systolic blood pressure $56 \mathrm{mmHg}$, pulse rate 96 beats per minutes, and respiratory rate 30 breaths per minute. The lungs were clear. Cardiopulmonary examination showed no cardiomegaly or heart murmur. Initial laboratory studies showed leukocyte cell count $23,300 \times 10^{4} / \mu 1$, hemoglobin $21.9 \mathrm{~g} / \mathrm{dl}$, hematocrit $61.7 \%$, CRP $0.10 \mathrm{mg} / \mathrm{dl}$, GOT $13 \mathrm{IU} / \mathrm{l}, \mathrm{GPT} 12 \mathrm{IU} / \mathrm{l}, \mathrm{LDH} 178$ IU/l, Ch-E 3,396 IU/l, blood urea nitrogen $25 \mathrm{mg} / \mathrm{dl}$, creatinine $1.67 \mathrm{mg} / \mathrm{dl}$, total protein $4.3 \mathrm{~g} / \mathrm{dl}$, albumin $2.5 \mathrm{~g} / \mathrm{dl}$, sodium $134 \mathrm{mEq} / l$, and potassium $5.0 \mathrm{mEq} / l$. The tests for antinuclear antibodies and antibodies to DNA were negative. Urine analysis for proteinuria and occult blood in the urine were negative. A blood gas analysis showed $\mathrm{pH} 7.224, \mathrm{PaO}_{2} 112.6 \mathrm{mmHg}$,

From the Department of Nephrology, Saitama Medical School, Saitama

Received for publication October 25, 2001; Accepted for publication June 10, 2002

Reprint requests should be addressed to Dr. Hiromichi Suzuki, the Department of Nephrology, Saitama Medical School, 38 Morohongo, Moroyama-machi, Iruma-gun, Saitama 350-0495 
$\downarrow \downarrow \underset{40}{\text { Methyl PSL Div } 1,000 \mathrm{mg} / \text { day }}$
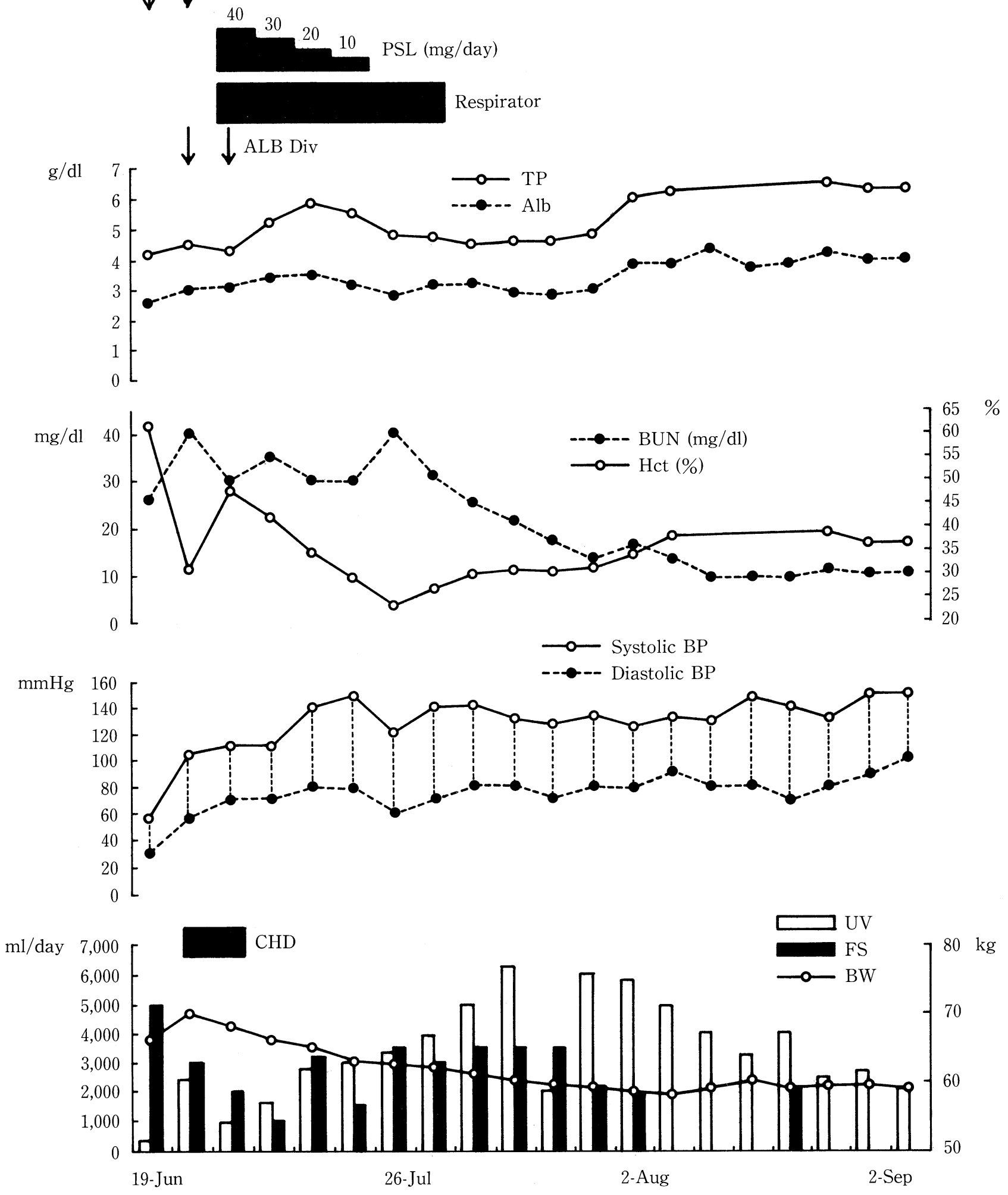

Figure 1. Clinical course of the present case. Div: intravenous drip infusion, CHD: continuous hemodialysis, ALB: albumin, PSL: prednisolone, BUN: blood urea nitrogen, Hct: hematocrit, TP: total protein, Systolic BP: systolic blood pressure, Diastolic BP: diastolic blood pressure, UV: urine volume, FS: fluid supplementation, BW: body weight. 
$\mathrm{PaCO}_{2} 25.7 \mathrm{mmHg}$, and $\mathrm{HCO}_{3}^{-} 10.4 \mathrm{mmol} / l$. All adrenal hormones were beyond the upper levels of the normal ranges. A chest radiograph revealed slight pleural effusion without abnormal shadows in the lungs. An echocardiogram did not show any signs of pericardial effusion. The left ventricular ejection fraction was $81 \%$ and wall motion was normal. The patient had a small amount of monoclonal IgG- $\kappa$ protein $(6.1 \mathrm{~g} / \mathrm{l})$ in his serum. Bence-Jones proteinuria was negative. We diagnosed his illness as hypovolemic shock accompanied by hypoproteinemia. On the 7th hospital day, bone marrow aspiration was carried out and was approximately normal. A total of $10 l$ of fluid and a $20 \mathrm{~g}$ dose of albumin were administered intravenously during the first 72 hours. On days 2 and 3, a 1,000 mg dose of hydrocortisone was administered intravenously. In spite of the disappearance of generalized edema, dyspnea started. A chest radiograph revealed congestion of the lung and blood gas analysis $\left(100 \% \mathrm{O}_{2} 10 \mathrm{l} / \mathrm{min}\right)$ showed $\mathrm{pH} 7.344, \mathrm{PaO}_{2} 28.7$ $\mathrm{mmHg}, \mathrm{PaCO}_{2} 46.0 \mathrm{mmHg}$ and $\mathrm{HCO}_{3}{ }^{-} 24.5 \mathrm{mmol} / l$. On the third hospital day, he was intubated and mechanical ventilation was maintained. A twenty gram dose of albumin was administered intravenously for one hour and then $60 \mathrm{mg}$ of furosemide was injected intravenously as a single dose. Because the urine volume was less than $1,000 \mathrm{ml}$ daily and dyspnea worsened, continuous extracorporeal ultrafiltration was performed and $80 \mathrm{ml} / \mathrm{h}$ of water removed for 48 hours from the third hospital day. From the fourth hospital day, urine output increased gradually and blood gas analysis showed improvement of pulmonary congestion. Mechanical ventilation was discontinued and the dose of furosemide was reduced to $20 \mathrm{mg}$ per day on the 9th hospital day. On the 7th hospital day, plasma cytokines showed the following values: interleukin-2 non-detectable (normal range $<0.8$ ), vascular endothelium growth factor (VEGF) $75 \mathrm{pg} / \mathrm{ml}(62-707 \mathrm{pg} / \mathrm{ml})$, complement-3a (C3a) 4,430 ng/ml (50-200 ng/ml), and complement-5a (C5a) nondetectable $(<10)$. The patient experienced a progressive reduction in his body weight and a resolution of the generalized edema. A chest radiograph showed no pleural effusion. On the 20th hospital day, gastrointestinal examination showed no evidence of gastritis, gastrointestinal ulcer or malignancy. From these data, we diagnosed his illness as SCLS. On the 50th hospital day, plasma cytokines including interleukin-2, VEGF, C3a, and C5a were all within normal levels. On the 57th hospital day, his body weight was $59 \mathrm{~kg}$ and laboratory findings showed total protein $6.8 \mathrm{~g} / \mathrm{dl}$, albumin $4.3 \mathrm{~g} / \mathrm{dl}$, blood urea nitrogen 12 $\mathrm{mg} / \mathrm{dl}$, and creatinine $0.80 \mathrm{mg} / \mathrm{dl}$ and a small monoclonal IgG$\kappa(10.0 \mathrm{~g} / l)$ protein was found in his serum. He was discharged from our hospital.

\section{Discussion}

We described a 46-year-old man who was hospitalized because of a 3-month history of recurrent generalized edema and hypotension accompanied by hypoproteinemia. The clinical and laboratory findings of our patient suggested strongly the diagnosis of SCLS. The detection of a serum monoclonal antibody, IgG- $\kappa$ paraprotein, also supports the diagnosis of SCLS. Dur- ing the capillary leakage phase, we infused fluid to maintain perfusion pressure for the kidneys, brain, heart and other vital organs. However, because of this process, an episode of acute pulmonary edema occurred on the 3rd hospital day. He was intubated and support with mechanical ventilation was begun. Continuous extracorporeal ultrafiltration was started. From these consequent events, acute pulmonary edema was produced by two mechanisms, the recruitment of the initially extravasated fluids and our supplementation of fluid.

SCLS is a rare condition characterized by recurrent episodes of generalized edema and severe hypotension, associated with unexpected episodic capillary high-permeability. Episodic attacks are characterized by a marked shift of plasma, up to $70 \%$, from the intravascular space to the extravascular space. This syndrome presents with a characteristic triad of hypotension, hemoconcentration, and hypoalbumiuria. The average age is 46 years (range, 9 to 69 years) and the male: female ratio is 1.2: 1 (35: 29). Only two cases of SCLS have been reported in Japan. The etiology of SCLS is unknown, although a possible role of viral infection has been proposed $(4,5)$. The presence of a serum monoclonal immunoglobulin in almost all patients initially was a focus of attention (2). The types of monoclonal immunoglobulin were IgG, 59 (IgG- $\kappa, 44$; IgG- $\lambda$, 10; unknown, $5)$ and $\operatorname{IgA}, 3(\operatorname{IgA}-\lambda, 3)$. Amoura et al (4) hypothesized that a monoclonal immunoglobulin could be responsible for endothelial damage and capillary hyperpermeability. Zhang et al (6) reported that paraproteins from patients with SCLS do not exert any cytotoxic effect, alone or in the presence of neutrophils, towards cultured human endothelial cells. In SCLS patients, numbers of mononuclear cells expressing the interleukin2 (IL-2) receptor have been reported to be increased markedly during the capillary leakage phase (7). Early activation of the classical pathway of complement has been reported in other cases (8). Interestingly, in our case, C3a was high but serum IL-2 was at a normal level on the 7th hospital day. On the 57th hospital day, C3a was within normal limits. From these data, there is a possibility that activation of the complement pathway played some role in the present case.

Multiple regimens, based on possible pathological mechanisms, have been tried with various degrees of success, including theophylline, terbutaline, salbutamol, steroids, diuretics, calcium antagonists, plasmapheresis and Gingko biloba extracts (9). Although morbidity and mortality rates associated with SCLS are high, the prognosis seems to have improved recently (4). Of the first 25 cases described, 19 (76\%) did not survive 5 years after the diagnosis of SCLS. Amoura et al (4) reported 13 cases of SCLS. Eight patients were still alive after a mean follow up of 5.6 years. He concluded that his series showed an improvement in the prognosis of SCLS attributable most likely to technical advances, including treatment with terbutaline, aminophylline, cyclosporine and intravenous immunoglobulins, and to careful monitoring of fluid volume with intensive care during attacks.

In SCLS, during the stable period, capillary permeability is normal. Extravasation occurs only during an episode of SCLS attack. It has been reported that the clinical features of an acute 


\section{Chinara et al}

episode of SCLS consist of two phases (10): The initial capillary leak phase may last from 1 to 4 days and is essentially a phase of acute hypovolemia attributable to marked extravasation of intravascular fluids and macromolecules. The capillaries are unable to retain macromolecules smaller than $200 \mathrm{kDa}$ and sometimes as large as $900 \mathrm{k} \mathrm{Da}(2)$. Laboratory findings reveal hemoconcentration, leukocytosis, increase in IgM, decrease in albumin, and decrease in $\operatorname{IgG}(6)$. At the peak of an SCLS episode, the clinical features include generalized edema, intestinal edema, ascites, pleural and pericardial effusions (10). In this phase, the cerebral, coronary and renal circulation are decreased because of hypovolemic shock. Rhabdomyolysis may be an important complication of SCLS (3). Acute renal failure can result from acute tubular necrosis because of hypovolemic shock accompanied by rhabdomyolysis (3). The recruitment phase follows the capillary leak phase and involves the normalization of the extraordinary vascular leakage, resulting in recruitment of the initially extravasated fluid and macromolecules. In the recruitment phase, intravascular overload may occur easily and result in acute pulmonary edema. In the present case, acute pulmonary edema developed on the 3rd hospital day and the patient was intubated and mechanical ventilation was performed. We speculate that intravascular overloading accompanied by the recruitment of the initially extravasated fluids and macromolecules resulted in acute pulmonary edema.

In the present case, we treated our patient with steroid pulse therapy in the acute capillary leak phase. Steroid administration may have a role during the capillary leak phase, when cytokine-mediated mechanisms of endothelial damage are prominent (10). It was shown that macromolecule leakage in response to various stimuli, including histamine and bradykinin, can be inhibited by pretreatment with $\beta_{2}$ stimulants, terbutaline-selective $\beta_{2}$ stimulant and isoprenaline (11). The combination of terbutaline and aminophylline therapy has been reported as a first line prophylactic therapy by Droder et al (12) and has been used by several authors $(9,10,12)$. Recently, Tahirkheli et al (10) reported that a regimen of terbutaline and theophylline seems to be effective prophylaxis against SCLS. Both terbutaline and theophylline are thought to interfere with capillary permeability and to antagonize the effects of cytokines or other mediators, inducing endothelial damage and vascular hyperpermeability. However, no efficacious pharmacological treatment has been clearly established.

We report a case of a SCLS. Intravascular overloading accompanied by the recruitment of the initially extravasated fluids and macromolecules resulted in acute pulmonary edema. Intensive care unit and careful monitoring of fluid volume are necessary in SCLS.

\section{References}

1) Clarkson B, Thompson D, Horwith M, Luckey H. Cyclical edema and shock due to increased capillary permeability. Am J Med 29: 193-216, 1960.

2) Atkinson JP, Waldmann TA, Stein SF, et al. Systemic capillary leak syndrome and monoclonal IgG gammopathy; studies in a sixth patient and a review of the literature. Medicine (Baltimore) 56: 225-239, 1977.

3) Dolberg-Stolik OC, Putterman C, Rubinow A, Rivkind AI, Sprung CL. Idiopathic capillary leak syndrome complicated by massive rhabdomyolysis. Chest 104: 123-126, 1993.

4) Amoura Z, Papo T, Ninet J, et al. Systemic capillary leak syndrome: report on 13 patients with special focus on course and treatment. Am J Med 103: 514-519, 1997.

5) Lassoued K, Clauvel JP, Similowski T, Autran B, Bengoufa D, Oksenhendler E. Pulmonary infections associated with systemic capillary leak syndrome attacks in a patient with hypogammaglobulinemia. Intensive Care Med 24: 981-983, 1998.

6) Zhang W, Ewan PW, Lachmann PJ. The paraproteins in systemic capillary leak syndrome. Clin Exp Immunol 93: 424-429, 1993.

7) Cicardi M, Gardinali M, Bisiani G, Rosti A, Allavena P, Agostoni A. The systemic capillary leak syndrome: appearance of interleukin-2 receptor positive cells during attacks. Ann Intern Med 113: 475-477, 1990.

8) Lofdahl CG, Solvell L, Laurell AB, Johansson BR. Systemic capillary leak syndrome with monoclonal IgG and complement alterations. Acta Med Scand 206: 405-412, 1979.

9) Teelucksingh S, Padfield PL, Edwards CR. Systemic capillary leak syndrome. Q J Med 75: 515-524, 1990.

10) Tahirkheli NK, Greipp PR. Treatment of the systemic capillary leak syndrome with terbutaline and theophylline. A case series. Ann Intern Med 130: 905-909, 1999.

11) Svensjo E, Persson CG, Rutili G. Inhibition of bradykinin induced macromolecular leakage from post-capillary venules by a beta2-adrenoreceptor stimulant, terbutaline. Acta Physiol Scand 101: 504-506, 1977.

12) Droder RM, Kyle RA, Greipp PR. Control of systemic capillary leak syndrome with aminophylline and terbutaline. Am J Med 92: 523-526, 1992. 